\title{
On Linearized Korteweg-de Vries Equations
}

\author{
Alfredo Villanueva \\ Department of Mathematical Sciences, University of Puerto Rico at Mayagüez \\ PO box 9000, Mayagüez PR 00681, USA
}

Tel: 1-787-832-4040 Ext 5878 E-mail: alfredo.villanueva@upr.edu

Received: September 13, 2011 Accepted: September 30, 2011 Published: February 1, 2012

doi:10.5539/jmr.v4n1p2 URL: http://dx.doi.org/10.5539/jmr.v4n1p2

\begin{abstract}
Korteweg-de Vries equations (KdV) provide a way of modeling waves on shallow water surfaces. These equations, begun by John Scott Russell in 1834 through observation and experiment, are a type of nonlinear differential equations. Originating with constant coefficients, they now include time-dependent coefficients, modeling ion-acoustic waves in plasma and acoustic waves on a crystal lattice, and there is even a connection with the Fermi-Pasta-Ulam problem. Most of the solutions are given by solitons or by numerical approximations. In this work we study a linearized KdV equation with time-dependent coefficients (including fifth-order $\mathrm{KdV}$ ) by using a special ansatz substitution.
\end{abstract}

Keywords: Linearized Kortewege Vries equations, Soliton-like solutions, Time-dependent coefficients

\section{Introduction}

Korteweg-de Vries equations are nonlinear dispersive partial differential equations and their solutions are what are called solitons (solitary waves). This solitary wave was first observed and studied by Scott Russell in 1834, and he describes his first encounter in (S. Russell, 1844):

"I believe I shall best introduce this phenomenon by describing the circumstances of my own first acquaintance with it. I was observing the motion of a boat which was rapidly moving along a narrow channel by a pair of horses, when the boat suddenly stopped-not so the mass of water in the channel which it had put in motion; it accumulated round the prow of the vessel in a state of violent agitation; then suddenly leaving it behind, rolled forward with great velocity, assuming the form of a large solitary elevation, a rounded, smooth and well defined heap of water, which continued its course along the channel apparently without change of form or dimension of speed. I followed it on horseback, and overtook it still rolling on at a rate of some eight or nine miles an hour, preserving its original figure some thirty feet long and a foot to foot and a half in height. Its height gradually diminished, and after a chase of one or two miles I lost it in the windings of the channel. Such, in the month of August 1834, was my first chance interview with that singular and beautiful phenomenon which I have called the Wave of Translation, a name which it now very generally bears."

Russell deduced empirically the velocity $c$ of this solitary wave by the formula $c^{2}=g(a+h)$, where $\mathrm{g}$ is gravity, a is the amplitude, and $\mathrm{h}$ is the depth of the water.

But it was Boussinesq and Lord Rayleigh who showed that the soliton $u(x, t)$ has the profile:

$$
\psi(x, t)=\operatorname{aSech}^{2}(\beta(x-c t)) .
$$

They didn't know this soliton could be expressed in a simple equation and it wasn't until (D. J. Korteweg, 1895) that Korteweg-de Vries deduced a nonlinear differential equation whose solution is the soliton (1).

A simplified version of this equation (after a variety of transformations) is

$$
\frac{\partial \psi}{\partial t}+\frac{\partial^{3} \psi}{\partial x^{3}}+6 \psi \frac{\partial \psi}{\partial x}=0
$$

with the solution

$$
\psi(x, t)=\frac{c}{2} \operatorname{Sech}^{2}\left(\frac{\sqrt{c}}{2}(x-c t)\right) .
$$

Now days this equation is called a Korteweg-de Vries equation (KDV equation) and has been intensively studied after Zabusky and Kruskal in (Zabusky, 1965) made a connection with an earlier work by Fermi, Pasta, and Ulam. Their 
observations of these wave solutions can interact with each other and after collision they retain their initial forms, which led Zabusky and Kruskal to name these solutions "solitons" (after proton, photon, etc.). For an introductory reading about solitons, see (Drazin, 1992).

The search for solutions of nonlinear differential equations has been a long journey and there still is not a unified method to solve all these equations. There are many methods to solve different types of nonlinear equations, such as the Inverse Scattering Transform (IST), one of the most important transformations after the Fourier Transform for nonlinear equations (Gardner, 1967); the Backlund Transformation, introduced by L. Bianchi and A. V. Backlund in the nineteenth century, which has its origins in differential geometry to essentially transform a surface in a pseudospherical surface as a solution of a sin-Gordon equation (Backlund, 1883; Bianchi, 1885; Bianchi, 1890; Bianchi, 1892); for a more modern treatment of this topic read (Rogers, 2002); bilinear transformation; the sine cosine method; the Riccati expansion method; Painleve analysis; Jacobi elliptic function; tanh and tanh extended methods; separation of variables; ansatz substitutions and more.

There is much work done on finding exact solutions for KDV and modified KDV equations, especially with constant coefficients. We would like to mention an interesting substitution, $\psi(x, t)=A \operatorname{Sech}^{2}[k(x-v t)]+\beta(t)$, has been applied to KDV, Bureger, Gadner and fifth-KDV equations with time-dependent coefficients in (Wazwas, 2010; Wazwas, 2010) and (A. Wazwas, Press).

The aim of this paper is to use an ansatz substitution to find soliton-like solutions of the linearized KDV and fifth-KDV equations with time-dependent coefficients. This equation itself has many applications. For example, it is usually used to study the propagation of fairly long waves in the shallow water (Witham, 1974).

Notice that KDV equation (1) and

$$
\frac{\partial \psi}{\partial t}+\frac{\partial^{3} \psi}{\partial x^{3}}-6 \psi^{2} \frac{\partial \psi}{\partial x}=0
$$

can both be linearized for the amplitude, which gives the equation

$$
\frac{\partial \psi}{\partial t}+\frac{\partial^{3} \psi}{\partial x^{3}}=0
$$

The motivation for this ansatz comes from (Cordero-Soto, 2008), where they developed a substitution

$$
\psi(x, y, t)=A(t) e^{i S(x, y, t)}
$$

with

$$
S(x, y, t)=\alpha(t) x^{2}+\beta(t) x y+\gamma(t) y^{2}+\delta(t) x+\varepsilon(t) y+\kappa(t)
$$

and

$$
A(t)=\frac{1}{\sqrt{2 \pi i \mu(t)}}
$$

for the time-dependent Schrödinger equation with the quadratic Hamiltonian of the form

$$
i \frac{\partial \psi}{\partial t}=-a(t) \frac{\partial^{2} \psi}{\partial x^{2}}+b(t) x^{2} \psi-\left(c(t) x \frac{\partial \psi}{\partial x}+d(t) \psi\right)-f(t) x \psi+i g(t) \frac{\partial \psi}{\partial x} .
$$

This substitution lead to an ordinary differential equations system that has been solved in (Cordero-Soto, 2008); they have used this ansatz in (Suazo, 2010; Suazo, 2011) where they work a quadratic and nonlinear quadratic Hamiltonian.

\section{On the Linearized of KdV Equations with Time-Dependent Coefficients}

As mentioned before we are working with an ansatz substitution which is a modified version of the one used in (CorderoSoto, 2008; Suazo, 2010; Suazo, 2011). The first thing to notice about their work is the splitting form of the solution $\psi(x, y, t)$ into $A(t)$ and $e^{S(x, y, t)}$, where $S(x, y, t)$ can be seen as a polynomial function on $x$ and $y$ with time-dependent coefficients.

To work on a Schrödinger equation will imply to differentiate $S(x, y, t)$ twice with respect to $x$, so adding the quadratic Hamiltonian (quadratic in $x$ ) could potentially compensate and balance a system of equations determined by setting the derivative of $S(x, y, t)$ twice with respect to $x$ equal to the derivative of $S(x, y, t)$ with respect to $t$.

With this in mind it is natural to ask, what about Hamiltonians of higher degrees? We have managed to have some results on a Schrödinger equation of fourth degree Hamiltonian with time-dependent coefficients, but with some additional condition on the coefficients. This work will appear somewhere else. We did this by increasing the degree of $S(x, y, t)$ (in the sense mentioned before). 
From these works, we realized that by increasing the derivative with respect to $x$ we can potentially compensate the system we get from this procedure, which lead us to linear KdV equations with time-dependent coefficients including the fifth order case.

For this work, we are considering the following differential equation:

$$
\frac{\partial \psi}{\partial t}=-a(t) \frac{\partial^{3} \psi}{\partial x^{3}}+b(t) \frac{\partial \psi}{\partial x}+c(t) \psi
$$

Notice that we can get rid of $c(t) \psi$ by changing $\mu=\psi e^{\int c(\tau) d \tau}$, so we can always consider the simplified version:

$$
\frac{\partial \psi}{\partial t}=-a(t) \frac{\partial^{3} \psi}{\partial x^{3}}+b(t) \frac{\partial \psi}{\partial x} .
$$

We only require that $a(t)$ and $b(t)$ be integrable functions.

\subsection{Strategy}

We then assume our solution to (11) as in (6)

where

$$
S(x, y, t)=\theta(t) y^{3}+\alpha(t) x^{2}+\beta(t) x y+\gamma(t) y^{2}+\delta(t) x+\varepsilon(t) y+\kappa(t) .
$$

Here we modified $S(x, y, t)$ by a cubic polynomial in $x$ and $y$ with time-dependent coefficients in order to compensate our following system from the term $\frac{\partial^{3} \psi}{\partial x^{3}}$.

Before doing a direct substitution, we first find the derivatives of $S(x, y, t)$ :

$$
\begin{aligned}
& \frac{\partial S}{\partial t}=\theta^{\prime}(t) y^{3}+\alpha^{\prime}(t) x^{2}+\beta^{\prime}(t) x y+\gamma^{\prime}(t) y^{2}+\delta^{\prime}(t) x+\varepsilon^{\prime}(t) y+\kappa^{\prime}(t) \\
& \frac{\partial S}{\partial x}=\beta(t) y+\delta(t) \\
& \frac{\partial^{2} S}{\partial x^{2}}=0 .
\end{aligned}
$$

Doing a substitution in equation (11) we end up with 2 equations:

$$
\begin{gathered}
A^{\prime}(t)=0 \\
A(t)=A \\
\frac{\partial S}{\partial t}=a(t)[\beta(t) y+\delta(t)]^{3}+b(t) \beta(t) y+b(t) \delta(t) .
\end{gathered}
$$

From equation (15) we get the following results:

$$
\begin{aligned}
& \beta(t)=\beta \\
& \theta(t)=\beta^{3} \int_{0}^{t} a(\tau) d \tau \\
& \delta(t)=\delta \\
& \gamma(t)=3 \beta^{2} \int_{0}^{t} a(\tau) \delta(\tau) d \tau \\
& \varepsilon(t)=\beta \int_{0}^{t}\left[3 a(\tau) \delta^{2}(\tau)+b(\tau)\right] d \tau \\
& \kappa(t)=\int_{0}^{t}\left[a(\tau) \delta^{3}(\tau)+b(\tau) \delta(\tau)\right] d \tau .
\end{aligned}
$$


From here we have explicitly our function $S(x, y, t)$, which defines our solution $\psi(x, y, t)$ of $(11)$. Notice we only require that $a(t)$ and $b(t)$ be integrable functions. Next we give some examples.

\subsection{Example 1}

If we consider that $a(t)=b(t)=1$, the equation (11) becomes

$$
\frac{\partial \psi}{\partial t}+\frac{\partial^{3} \psi}{\partial x^{3}}=0
$$

From our previous section

$$
A(t)=A
$$

$$
\begin{aligned}
& \beta(t)=\beta \\
& \theta(t)=\beta^{3} \int_{0}^{t} a(\tau) d \tau=\beta^{3} t \\
& \delta(t)=\delta \\
& \gamma(t)=3 \beta^{2} \delta \int_{0}^{t} a(\tau) d \tau=3 \delta \beta^{2} t \\
& \varepsilon(t)=3 \beta \delta^{2} \int_{0}^{t} a(\tau) d \tau=3 \beta \delta^{2} t \\
& \kappa(t)=\delta^{3} \int_{0}^{t} a(\tau) d \tau=\delta^{3} t,
\end{aligned}
$$

so the solution is

$$
\psi(x, y, t)=A e^{i\left(\beta^{3} y^{3} t+\beta x y+3 \delta \beta^{2} y^{2} t+\delta x+3 \beta \delta^{2} y t+\delta^{3} t\right)}
$$

where $\beta$ and $\delta$ are constants. Notice that if we consider $\delta=0$ and $\beta=2 \pi$, we get the classical solution usually found by the Fourier transform,

$$
\psi(x, y, t)=A e^{i\left(8 \pi^{3} y^{3} t+2 \pi x y\right)} .
$$

\subsection{Example 2}

If we consider $a(t)=\operatorname{cost}, b(t)=0$ and $c(t)=3 t^{2}$, the equation (10) becomes

$$
\frac{\partial \psi}{\partial t}=-\cos t \frac{\partial^{3} \psi}{\partial x^{3}}+3 t^{2} \psi
$$

From our previous section,

$$
\begin{aligned}
& A(t)=e^{t^{3}} \\
& \beta(t)=\beta_{o} \\
& \theta(t)=\beta^{3} \int_{0}^{t} \cos (\tau) d \tau=\beta^{3} \sin t \\
& \delta(t)=\delta_{o} \\
& \gamma(t)=3 \delta \beta^{2} \sin t \\
& \varepsilon(t)=3 \beta \delta^{2} \sin t \\
& \kappa(t)=\delta^{3} \sin t,
\end{aligned}
$$

so the solution is

$$
\psi(x, y, t)=e^{t^{3}} e^{i\left(\beta^{3} y^{3} \sin t+\beta x y+3 \delta \beta^{2} y^{2} \sin t+\delta x+3 \beta \delta^{2} y \sin t+\delta^{3} \sin t\right)}
$$

with $\beta$ and $\delta$ as constants. If $\delta=0$, then 


$$
\psi(x, y, t)=e^{t^{3}+i\left(\beta^{3} y^{3} \sin t+\beta x y\right)}
$$

\section{Linearized Fifth-Order KdV Equation}

In this section we apply the substitution discussed before to the following fifth-order KdV:

$$
\frac{\partial \psi}{\partial t}=-a(t) \frac{\partial^{5} \psi}{\partial x^{5}}+b(t) \frac{\partial^{3} \psi}{\partial x^{3}}
$$

Let our solution be as in (6), where

$$
S(x, y, t)=\alpha(t) y^{5}+\beta(t) y^{4}+\gamma(t) y^{3}+\delta(t) x y+\varepsilon(t) y^{2}+\kappa(t) x+\theta(t) y+\omega(t) .
$$

Again in this technique the key element is to get the right $S(x, t)$; in this case we found that fifth degree in $y$ and at most first degree on $x$ will give us a solvable system of equations.

Derivatives of $S(x, y, t)$ are as follows:

$$
\begin{aligned}
& \frac{\partial S}{\partial t}=\alpha^{\prime}(t) y^{5}+\beta^{\prime}(t) y^{4}+\gamma^{\prime}(t) y^{3}+\delta^{\prime}(t) x y+\varepsilon^{\prime}(t) y^{2}+\kappa^{\prime}(t) x+\theta^{\prime}(t) y+\omega^{\prime}(t) \\
& \frac{\partial S}{\partial x}=\delta(t) y+\kappa(t) \\
& \frac{\partial^{2} S}{\partial x^{2}}=\frac{\partial^{3} S}{\partial x^{3}}=\frac{\partial^{4} S}{\partial x^{4}}=\frac{\partial^{5} S}{\partial x^{5}}=0 .
\end{aligned}
$$

Also we have

$$
\begin{gathered}
\frac{\partial \psi}{\partial t}=A^{\prime}(t) e^{i S(x, y, t)}+i A(t) e^{i S(x, y, t)} \frac{\partial S}{\partial t} \\
\frac{\partial^{3} \psi}{\partial x^{3}}=A(t) e^{i S(x, y, t)}\left[i \frac{\partial^{3} S}{\partial x^{3}}-3 \frac{\partial^{2} S}{\partial x^{2}} \frac{\partial S}{\partial x}-i\left(\frac{\partial S}{\partial x}\right)^{3}\right] \\
=-i A(t) e^{i S(x, y, t)}(\delta(t) y+\kappa(t))^{3} \\
\frac{\partial^{5} \psi}{\partial x^{5}}=\quad A(t) e^{i S(x, y, t)}\left[i \frac{\partial^{5} S}{\partial x^{5}}-10 \frac{\partial^{2} S}{\partial x^{2}} \frac{\partial^{3} S}{\partial x^{3}}-5 \frac{\partial^{4} S}{\partial x^{4}} \frac{\partial S}{\partial x}-15 i\left(\frac{\partial^{2} S}{\partial x^{2}}\right)^{2}\right. \\
\left.=-10 i \frac{\partial^{3} S}{\partial x^{3}}\left(\frac{\partial S}{\partial x}\right)^{2}+10 \frac{\partial^{2} S}{\partial x^{2}}\left(\frac{\partial S}{\partial x}\right)^{3}+i\left(\frac{\partial S}{\partial x}\right)^{5}\right] \\
=-i A(t) e^{i S(x, y, t)}(\delta(t) y+\kappa(t))^{5} .
\end{gathered}
$$

So, replacing (31) in (26) we get

$$
A(t)=A
$$




$$
\begin{aligned}
& \alpha(t)=-\left(\delta_{o}\right)^{5} \int_{0}^{t} a(\tau) d \tau \\
& \beta(t)=-5\left(\delta_{o}\right)^{4} \kappa_{o} \int_{0}^{t} a(\tau) d \tau \\
& \gamma(t)=-10\left(\delta_{o}\right)^{3}\left(\kappa_{o}\right)^{2} \int_{0}^{t} a(\tau) d \tau-\left(\delta_{o}\right)^{3} \int_{0}^{t} b(\tau) d \tau \\
& \delta(t)=\delta_{o} \\
& \varepsilon(t)=-10\left(\delta_{o}\right)^{2}\left(\kappa_{o}\right)^{3} \int_{0}^{t} a(\tau) d \tau-3\left(\delta_{o}\right)^{2} \kappa_{o} \int_{0}^{t} b(\tau) d \tau \\
& \kappa(t)=\kappa_{o} \\
& \theta(t)=-5 \delta_{o}\left(\kappa_{o}\right)^{4} \int_{0}^{t} a(\tau) d \tau-3 \delta_{o}\left(\kappa_{o}\right)^{2} \int_{0}^{t} b(\tau) d \tau \\
& \omega(t)=-\left(\kappa_{o}\right)^{5} \int_{0}^{t} a(\tau) d \tau-\left(\kappa_{o}\right)^{3} \int_{0}^{t} b(\tau) d \tau .
\end{aligned}
$$

This defines the solution $\psi(x, y, t)$ of (26), and we only require that $a(t)$ and $b(t)$ be integrable functions too.

\subsection{Example}

If we consider $a(t)=b(t)=1$, the equation (26) becomes

$$
\frac{\partial \psi}{\partial t}=-\frac{\partial^{5} \psi}{\partial x^{5}}+\frac{\partial^{3} \psi}{\partial x^{3}}
$$

so a solution is

$$
\begin{aligned}
\psi(x, y, t)= & A(t) e^{i\left(-\delta_{o}^{5} y^{5} t-5\left(\delta_{o}\right)^{4} \kappa_{o} y^{4} t-\left[10\left(\delta_{o}\right)^{3}\left(\kappa_{o}\right)^{2}+\left(\delta_{o}\right)^{3}\right] y^{3} t+\delta_{o} x y-\left[10\left(\delta_{o}\right)^{2}\left(\kappa_{o}\right)^{3}+3\left(\delta_{o}\right)^{2} \kappa_{o}\right] y^{2} t\right)} \\
& e^{i\left(\kappa_{o} x-\left[5 \delta_{o}\left(\kappa_{o}\right)^{4}+3 \delta_{o}\left(\kappa_{o}\right)^{2}\right] y t-\left[\left(\kappa_{o}\right)^{5}+\left(\kappa_{o}\right)^{3}\right] t\right)} .
\end{aligned}
$$

\section{Conclusion}

In this paper we have seen an alternative way to find a solution of Linearized $\mathrm{KdV}$ equations via a modified ansatz substitution. We believe that by modifying $S(x, y, t)$, this method can be applied to other types of linear and nonlinear partial differential equations with time-dependent coefficients.

We also have noticed here, by analyzing how $S(x, y, t)$ has changed in (12) and (27), we can add extra terms in the equation so that our $\mathrm{KdV}$ increases in complexity but can still be solved by just modifying $S(x, y, t)$.

We observed that this technique will not be successful in general nonlinear differential equations, but in (E. Suazo, et al, 2010) the authors applied it to a nonlinear case. The nonlinear term has the form $|\psi(x, y, t)|^{2} \psi(x, y, t)$. Then using $\psi(x, y, t)=A(t) e^{i S(x, y, t)}$ the norm will cancel out the exponential square, so after that we can get a manageable ODE system. This observation gives us a clear conclusion about which nonlinear PDEs can be solved in this fashion, namely those which after substitution of the powers of exponentials can be simplified to first power. For instance we have successfully applied this strategy to a nonlinear Chiral equation from (Biswas, 2010).

\section{References}

Backlund, A. V. (1883). Om ytor med konstant negativ krkning. Lunds Universitets Åsskrift, 19, 1-48.

Bianchi, L. (1885). Sopra i sistemi tripli ortogonali di Weingarten. Annali di Matematica, 13, 177-234.

Bianchi, L. (1890). Sopra alcone nuove classi di superficie e di sistemi tripli ortogonali. Annali di Matematica, 18, 301-358.

Bianchi, L. (1892). Sulla traformazione di Backlund per le superficie pseudosferiche. Rendiconti Lincei, 5, 3-12.

Biswas, A. (2010). Chiral Solitons With Time - Dependent Coefficients. Int. J. Theor. Phys., 49, 79-83. http://dx.doi.org/10.1007/s10773-009-0180-1

Cordero-Soto, R. M. Lopez, E. Suazo, \& Suslov S. K. (2008). Propagator of a Charged Particle with a Spin in Uniform Magnetic and Perpendicular Electric Fields. Letters in Mathematical Physics, 84(2-3), 159-178. 
http://dx.doi.org/10.1007/s11005-008-0239-6

Drazin, P. G., \& Jhonson, R. S. (1992). Solitons: an introduction. New York: Cambridge Text in Applied Mathematics.

El-Wakil, S. A., Abdou A., \& Elhanbaly. (2006). New solitons and periodic wave solutions for nonlinear evolution equations. Physics Letters A, 353, 40-47. http://dx.doi.org/10.1016/j.physleta.2005.12.055

Gardner, C. S., Greene, J. M., Kruskal, M. D., \& Miura, R. M. (1967). Method for Solving the Korteweg-de Vries Equation. Physical Rev. Lett., 19, 1095-1097. http://dx.doi.org/10.1103/PhysRevLett.19.1095

Korteweg, D. J., \& de Vries, G. (1895). On the Change of Form of Long Waves Advancing in a Rectangular Canal, and on a New Type of Long Stationary Waves. Philosophical Magazine, 39, 240, 422-443. http://dx.doi.org/10.1080/14786449508620739

Nishino, A., Umeno, Y., \& Wadati, M. (1998). Chiral Nonlinear Schrdinger Equation. Chaos, Solitons and Fractals, 9, 7, 1063-1069. http://dx.doi.org/10.1016/S0960-0779(97)00184-7

Palais Richard S. (1997). The Symmetries of Solitons. Bull. Amer. Math. Soc, 34, 339-403. http://dx.doi.org/10.1090/S0273-0979-97-00732-5

Rogers, C., Schief, W. K., Backlund, \& Darboux. (2002). Transformations, Geometry and Moderns Applications in Soliton Theory. Cambridge University Press: Cambridge Texts in Applied Mathematics.

Russell Scott. (1844). Report on waves. Fourteenth meeting of the British Association for the Advancement of Science..

Suazo, E., \& Suslov, S. (2010). Soliton-like Solutions for Nonlinear Schrodinger Equation with Variable Quadratic Hamiltonians. [Online] Available: http://arxiv.org/PScache/arxiv/pdf/1010/1010.2504v4.pdf (November 24, 2010).

Suazo, E., Suslov, S. K., \& Vega-Guzman, J. M. (2011). The Riccati Equation and a Diffusion-type Equation. New York J. Math., 17a, 225-244.

Wazwas, A. (2010). Soliton Solutions for the Fifth-Order KdV Equation and the Kawahara Equation with time-Dependent Coefficients. Physica Scr, 82, 3, 035009. http://dx.doi.org/10.1088/0031-8949/82/03/035009

Wazwas, A., \& Triki, H. (2010). Bright Soliton solution to a Generalized Burgers-KdV Equation with Time-Dependent Coefficients. Appl. Math. Comput., 217, 466-471. http://dx.doi.org/10.1016/j.amc.2010.05.078

Wazwas, A. (2010). A Study on KdV and Gardner Equations with Time-Dependent Coefficients and Forcing Terms. Appl. Math. Comput. In Press.

Witham, G. B. (1974). Linear and nonlinear waves. New York: Wiley.

Zabusky, N. J., \& Kruskal, M. D. (1965). Interactions of Solitons in a Collisionless Plasma and the Recurrence of Initial States. Phys. Rev. Lett., 15, 240. http://dx.doi.org/10.1103/PhysRevLett.15.240 